\title{
Nickel discretization and quality review in Gllavica mine, Kosovo
}

\author{
Rafet Zeqiri ${ }^{1 * \otimes}$ (1) \\ ${ }^{1}$ University of Mitrovica, Mitrovica, 40000, Kosovo \\ *Corresponding author: e-mail rafet.zeqiri@ umib.net, tel. +38328530446
}

\begin{abstract}
Purpose. Professional approach to mineral deposit evaluation and estimation of Ni is done by modeling the ore body presenting the interest zone to be exploited.

Methods. With the intensive development of professional software dedicated to solving mining problems and ore reserves, Gllavica mine has implemented Surpac v.6.2 software. This software makes it possible to evaluate and estimate mineral deposit of $\mathrm{Ni}$ in Kosovo.

Findings. Based on geological exploration, drilling and sampling It was possible to find the quality of the ore, the footwall and hanging wall contact with ore body. The ore grade was examined and determined in the case of longitudinal geological profiles using Surpac v.6.2 Software by digitizing each drilling hole based on a database which was created considering assay, collar and survey files.

Originality. The use of appropriate software, creation of databases according to the values acquired from the field research provides the best possible assessment argued in this paper.

Practical implications. Geological research provides safety during ore exploitation that must be continuous throughout the exploitation phase. Geological research excludes blocks that differ from other blocks in terms of ore quality, so discretization of the source has enabled us to find the real quality and quantity of ore by comparing it with the profile method.
\end{abstract}

Keywords: mine, nickel, ore grade, exploitation, modeling

\section{Introduction}

This study encompasses the two research stages of "Gllavica" source, which both in the sense of the resources assessment and the exploitation plan are related to each other not only by the physical proximity and geologic connection but also because a good exploitation plan requires a primary homogenization from the exploitation stage of the source [1].

The use of modern computer software in cases requiring the primary homogenization of the source has proven to be a strong modern tool in the hands of the operating engineer. Pursuant to the presented concept in the process of ore exploitation from this source it is planned to assess the blocks from the existing field - stage one over the 574 meters quota, and the ore blocks from the new field - the so called second stage under the 574 meters quota [1], [2].

It is important that mine planning is preceded by detailed geological research by determining the depth and thickness of the nickel ore body, the other geological units surrounding deposit.

By means of geological drilling, the clay intrusions inside the source are determined. Their clay intrusions create ore depletion ending the modernization phase [1], [3]. To cor- rectly assess the quality and nickel reserves at the deposit, we apply advanced geostatistical methods in the assessment of the deposit by dividing (discrediting) the entire size of the mineral resource into $25 \times 25 \mathrm{~m}$ mini-blocks.

The SURPAC Software is highly advanced and flexible in handling and solving various problems in surface and underground mines by enabling us to create real 3D models of ore bodies that are defined through deep geologic drills. With the SURPAC software a professional 3D engineering design can be prepared based on the study data and geologic materials based on the quality of metals in deep drill cores. Through the SURPAC, mine engineers complete the geological model legends, geological maps, exploitation plans and various geostatistical information. SURPAC enables direct communication with the Civil 3D AutoCAD software thus facilitating the engineers' job in modelling and creating real terrain topographies and creating various geological profiles.

SURPAC provides powerful features and assists mine engineers in assessing the quality of metals at the source and the correct production planning at optimum cost.

Deep geological drillings dedicated to each mini-block enables the creation of three main databases (files) in Surpac 
software: Assay.csv, Collar.scv and Surve. Csv [4], [5]. The Assay.csv file contains the source quality records for each meter of drilling depth, describes the lithology of the geological contacts that meet the drilling as well as the quality of the accompanying metals extracted through the drilling rigs. The Collar.scv file contains the total depth of each drill as well as the geological drilling coordinates, while the Surve file. csv contains the name of the drill, the depth of each drill, the drill bit as well as the drill azimuth. With the help of geodetic surveys of the terrain topography we apply the professional software AutoCAD Civil 3D, creating the terrain situation with isohypses, the calculation of the exploitable reserves and the geological ones. Surpac software and AutoCAD Civil 3D exchange information about the source from the same databases, enabling us to evaluate the source correctly with the most modern geostatistical methods, knowing the quality of the ores and the volume of the ore, as well as knowing all the geological conditions surrounding the source [1].

\section{Materials and methods}

\subsection{Source description}

The "Gllavica" source of nickel silicate ore is characterized by several favorable factors in relation to the ore exploitation, the most important being the drop and extent of ore bodies located in relatively shallow depths from the surface. on the other hand, the ore, the accompanying rock and the cover are characterized by the favorable geoengineering conditions [6]-[8].

The source is researched with a $25 \times 25$ meters drilling grid, and the second stage was initially researched with a $25 \times 25$ meters drilling grid, to move on to a widened grid of $50 \times 50 \mathrm{~m}$ in the lower part of the source [1], [4]. The ore rich field of Gllavica mine is physical in the sense of the separating "border" between them, however, seen from the practical sense, the ore body is an entirety that belongs to the "breaking of the crust" with a defined extent and drop [4], [5], [9]-[11].

In the geological space of this stage, the research drills were initially conducted according to the $25 \times 25$ meters grid density, in a research length of $125 \mathrm{~m}$, to then move to the $50 \times 50 \mathrm{me}-$ ters drilling grid. The field contour-geologic border is defined based on the results from the conducted drills [1], [4], [5].

\subsection{Source presentation through isohypses}

From the drills conducted in the field, 579 drills in total, with the use of the appropriate software the field quota data and their sorting and interpolation within the border have been processed, and the field isohypses have been presented in (Fig. 1). The highest point of the field included within the borders of the source is 632.78 meters.

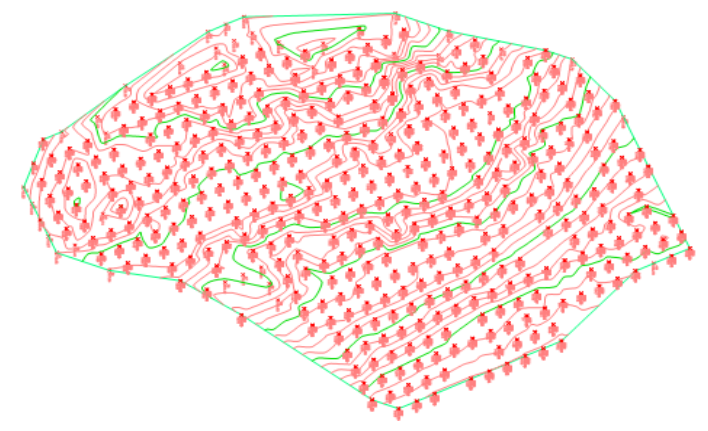

Figure 1. Isohypses of the initial configuration of the Gllavica mine area
Through the drills conducted in the field the upper contact of the ore body with the cover has been identified and this contact has been identified based on the zonal structural changes thus identifying the ore body ceiling (Fig. 2).

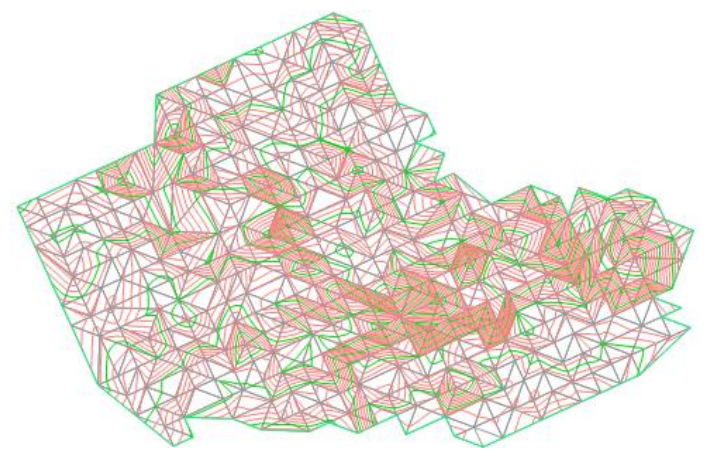

Figure 2. Isohypses of the ore body, hangingwall and footwall contact with the top soil

Similarly, from the field drills (508 drills in total), the zonal changes quota that determine the lower border of the minerals have been identified and this border presents the ore body floor (Fig. 3).

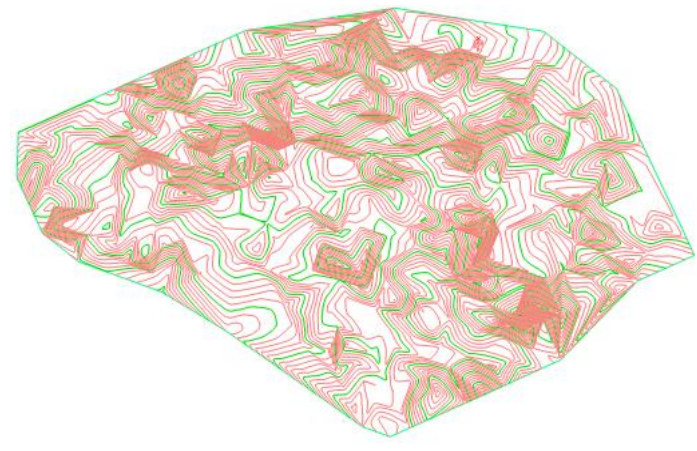

Figure 3. Ore body contact, footwall isohypses

Below, Figure 4 presents the nickel ore body, the source ceiling and floor contact.

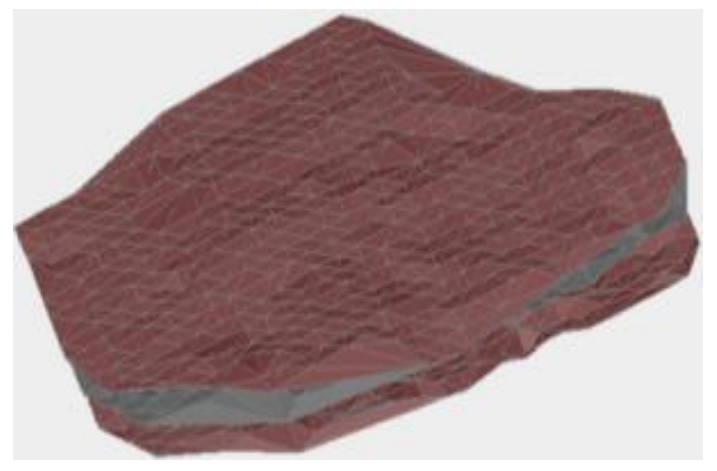

Figure 4. 3D presentation of the nickel mineral body between the geological contacts of the hangingwall and footwall

The use of modern computer software AutoCad Civil and specialized mining software SURPAC 6.2 has enabled the presentation of the condition of source position in Gllavica mine.

\subsection{Nickel ore exploitation according to levels}

The nickel ore exploitation in "Gllavica" mine is planned with the existing technology at the level height of 7 meters, 
whereas the ore will be transported with trucks to the existing deposit through the road created for ore transportation north of the mine, whereas the sterile is also transported by trucks and is deposited in the existing deposit. The ore and sterile exploitation dynamic and final condition for each level has been presented initially during the study in transverse west-east profile and the map the respective annex according to the level [11]-[13]. Each transverse profile contains the topography, drill number, ore body extent with the accompanying parameters defined by geological drills and other characteristics.

In this stage the following work stages according to block levels have been projected: first level $595 \mathrm{~m}$ quota, second level 588 meters quota, third level 581 meters quota and fourth level 595 meters quota (Fig. 5).

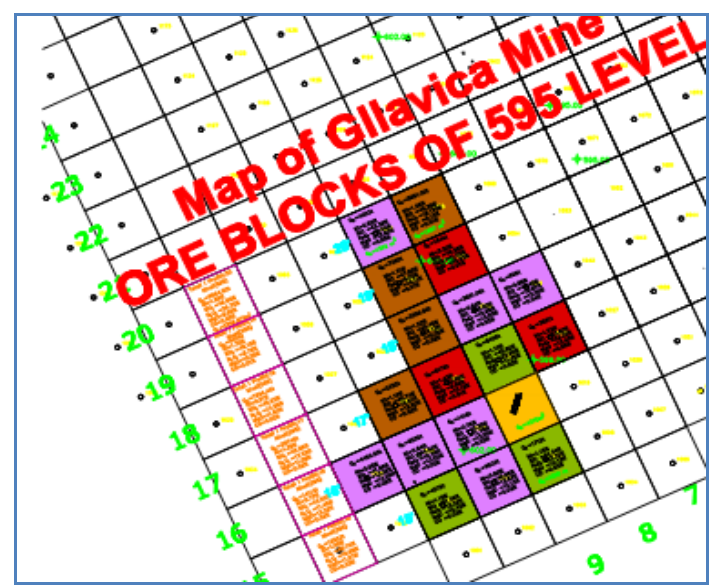

Figure 5. Blocks planned for extraction according to the nickel quality in each block

Figure 5 clearly shows the blocks and transverse profiles P-L 15-15 up to 20-20', and (Table 1) contains the 595 meters block parameters according to the profiles.

Tables 2-4 show the blocks and transverse profiles including the ore and sterile amounts and quality in (\%).

Average nickel percentage and accompanying components: $\mathrm{Ni}=1.06 \%, \quad \mathrm{SiO}_{2}=50.25 \%, \quad \mathrm{Fe}_{2} \mathrm{O}_{3}=19.00 \%$, $\mathrm{MgO}=15.14 \%, \mathrm{Co}=0.012 \%$.

Average nickel percentage and accompanying components: $\mathrm{Ni}=1.12 \%, \quad \mathrm{SiO}_{2}=46.22 \%, \quad \mathrm{Fe}_{2} \mathrm{O}_{3}=14.20 \%$, $\mathrm{MgO}=11.40 \%, \mathrm{Co}=0.05 \%$. Figure 6 show the entire mine zone divided in mini-blocks and the stretch of transverse profiles.

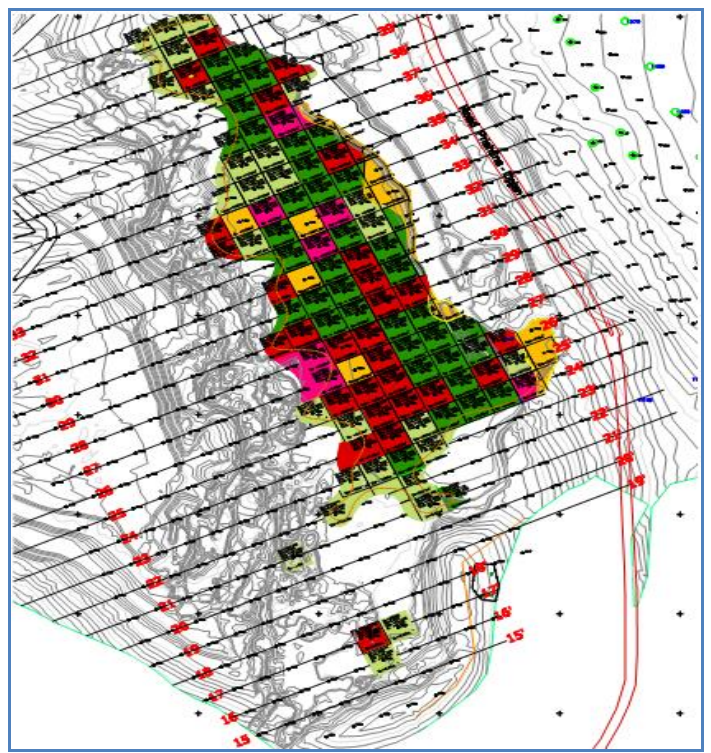

Figure 6. Transverse geological profiles throughout the research area including discrete blocks

Average nickel percentage and accompanying components: $\mathrm{Ni}=1.36 \%, \quad \mathrm{SiO}_{2}=40.54 \%, \quad \mathrm{Fe}_{2} \mathrm{O}_{3}=20.07 \%$, $\mathrm{MgO}=9.73 \%, \mathrm{Co}=0.037 \%$. Average nickel percentage and accompanying components: $\mathrm{Ni}=1.34 \%, \quad \mathrm{SiO}_{2}=46.60 \%$, $\mathrm{Fe}_{2} \mathrm{O}_{3}=23.31 \%, \mathrm{MgO}=12.06 \%, \mathrm{Co}=0.04 \%$.

Taking into account the deep geological research and the division of the source into miniblocks, it has been possible to correctly ascertain and evaluate the shape of the nickel ore body, the thickness of the ore body and the thickness of the cover on the ore body as well as the natural intrusions of clay inside the nickel source [4].

Transverse geological profiles (profiles 16 to profiling 42) have reflected the prospective mineralization area with higher quality of nickel and other accompanying elements, also through transverse profiles it has been ascertained that the thickness of the top cover over the nickel source ranges from 4 to $18 \mathrm{~m}$, while the nickel ore body is found in an irregular shape with a thickness of 4 to $40 \mathrm{~m}$.

Based on the geological profiles and the size of the blocks $25 \times 25 \mathrm{~m}$, the volumes of nickel ore, the volumes of the clay cover have been calculated and the quality of nickel and other accompanying elements from profile 16 to profile 42 has been determined according to the terrain quotas 558, 581 and $574 \mathrm{~m}$ (Tables 1-4).

Table 1. Block parameters for the 588 meters level

\begin{tabular}{|c|c|c|c|c|c|c|c|c|}
\hline \multirow{2}{*}{ Profiles } & \multirow{2}{*}{ Ore amount, $\mathrm{m}^{3}$} & \multirow{2}{*}{ Ore amount, $\mathrm{t}$} & \multirow{2}{*}{ Sterile amount, $\mathrm{m}^{3}$} & \multicolumn{5}{|c|}{ Quality at source, \% } \\
\hline & & & & $\mathrm{Ni} \%$ & $\mathrm{SiO}_{2}$ & $\mathrm{Fe}_{2} \mathrm{O}_{3}$ & $\mathrm{MgO}$ & $\mathrm{CO}$ \\
\hline 16 & 2843.749 & 5204.06 & 6218 & 0.62 & 43.67 & 9.48 & 27.18 & 0.01 \\
\hline 17 & 9324.158 & 17063.21 & 0.0 & 1.60 & 48.08 & 28.24 & 25.25 & 0.02 \\
\hline 18 & 4000.727 & 7321.33 & 0.0 & 1.01 & 42.86 & 12.65071 & 12.50 & 0.02 \\
\hline 19 & 8709.552 & 15938.48 & 3009.0 & 1.02 & 41.61 & 25.14 & 11.47 & 0.01 \\
\hline 20 & 19499.91 & 35684.83 & 2362 & 1.11 & 43.52 & 14.95 & 15.40 & 0.01 \\
\hline 21 & 18867.61 & 34527.72 & 3443.0 & 0.99 & 43.52 & 20.13 & 15.67 & 0.01 \\
\hline 22 & 15218.75 & 27850.32 & 1875 & 0.91 & 51.09 & 16.06 & 18.47 & 0.01 \\
\hline 23 & 6950 & 12718.5 & 2187 & 1.24 & 48.54 & 22.63 & 17.59 & 0.00 \\
\hline 24 & 6468.754 & 11837.82 & 2593 & 0.89 & 61.47 & 21.64 & 8.80 & 0.00 \\
\hline 25 & 10718.75 & 19615.32 & 0.0 & 0.77 & 61.03 & 16.09 & 9.57 & 0.02 \\
\hline 26 & 8031.246 & 14697.18 & 1562 & 0.77 & 61.03 & 16.09 & 9.57 & 0.02 \\
\hline 27 & 5912.497 & 10819.87 & 4806 & 1.23 & 58.96 & 16.72 & 11.10 & 0.01 \\
\hline 28 & 10181.25 & 18631.68 & 0.0 & 1.41 & 54.8 & 22.89 & 15.20 & 0.02 \\
\hline Total & 126727 & 231910.3 & 28055 & 1.06 & 50.25 & 19.0 & 15.14 & 0.012 \\
\hline
\end{tabular}


Table 2. Block parameters for the 581 meters level

\begin{tabular}{ccccccccc}
\hline \multirow{2}{*}{ Profiles } & \multirow{2}{*}{ Ore amount, $\mathrm{m}^{3}$} & \multirow{2}{*}{ Ore amount, $\mathrm{t}$} & \multirow{2}{*}{ Sterile amount, $\mathrm{m}^{3}$} & \multicolumn{4}{c}{ Quality at source, $\%$} \\
\cline { 5 - 8 } 16 & 4375 & 8006.25 & 331.25 & 770.84 & 47.84 & 12.66 & 13.12 & 0 \\
17 & 4375 & 8006.25 & 0 & 1.21 & 34.29 & 54.95 & 3.81 & 0 \\
19 & 2212.5 & 4048.875 & 0 & 1.16 & 47.66 & 30.74 & 9.60 & 0.040 \\
21 & 6000 & 10980 & 0 & 1.01 & 42.14 & 26.24 & 26.24 & 0.015 \\
22 & 3312.5 & 6061.875 & 750 & 0.83 & 44.82 & 5.84 & 5.84 & 0.042 \\
23 & 5312.5 & 9721.875 & 3750 & 0.97 & 45.13 & 22.64 & 19.92 & 0.031 \\
24 & 25875.01 & 47351.26 & 21343 & 1.38 & 30.01 & 20.75 & 6.86 & 0.034 \\
25 & 28062.5 & 51354.38 & 2287.5 & 1.12 & 33.73 & 18.00 & 14.55 & 0.024 \\
26 & 18375 & 33626.25 & 687.5 & 1.40 & 34.42 & 27.10 & 12.25 & 0.040 \\
27 & 13375 & 24476.25 & 5000 & 1.81 & 47.92 & 25.29 & 9.27 & 0.050 \\
28 & 18750 & 34312.5 & 7000 & 1.30 & 46.13 & 19.44 & 12.70 & 0.060 \\
29 & 19812.5 & 36256.88 & 12906.25 & 1.33 & 44.15 & 25.76 & 8.05 & 0.050 \\
30 & 21125 & 38658.75 & 750 & 1.62 & 48.74 & 20.80 & 9.35 & 0.050 \\
31 & 15312.5 & 28021.88 & 2750 & 1.19 & 30.04 & 21.76 & 6.90 & 0.040 \\
32 & 16625 & 30423.75 & 10368.75 & 1.66 & 27.04 & 6.43 & 4.69 & 0.010 \\
33 & 13937.5 & 25505.63 & 9312.5 & 1.23 & 58.77 & 19.37 & 6.42 & 0.090 \\
34 & 17750 & 32482.5 & 5937.5 & 1.22 & 35.24 & 11.18 & 5.95 & 0.01 \\
35 & 9375 & 17156.25 & 354.75 & 1.53 & 34.32 & 13.64 & 7.57 & 0.020 \\
36 & 7937.5 & 14525.63 & 6131.125 & 1.75 & 49.18 & 25.86 & 8.07 & 0.05 \\
37 & 5750 & 10522.5 & 3250 & 1.78 & 52.93 & 14.83 & 15.99 & 0.010 \\
38 & 4687.503 & 8578.13 & 5000 & 1.57 & 34.64 & 22.56 & 10.66 & 0.040 \\
39 & 7687.508 & 14068.14 & 1250 & 1.53 & 41.85 & 28.71 & 10.46 & 0.07 \\
40 & 5470.973 & 10011.88 & 0.0 & 1.73 & 60.48 & 11.13 & 14.69 & 0.020 \\
41 & 3262.809 & 5970.94 & 0.0 & 1.01 & 50.74 & 17.22 & 7.94 & 0.050 \\
42 & 4943.65 & 9046.88 & 3625 & 0.78 & 56.45 & 14.44 & 14.97 & 0.010 \\
Total & 283702.5 & 519175.5 & 102785.1 & 1.36 & 40.54 & 20.07 & 9.73 & 0.037 \\
\hline
\end{tabular}

Table 3. Block parameters for the 574 meters level

\begin{tabular}{|c|c|c|c|c|c|c|c|c|}
\hline \multirow{2}{*}{ Profiles } & \multirow{2}{*}{ Ore amount, $\mathrm{m}^{3}$} & \multirow{2}{*}{ Ore amount, $\mathrm{t}$} & \multirow{2}{*}{ Sterile amount, $\mathrm{m}^{3}$} & \multicolumn{5}{|c|}{ Quality at source, $\%$} \\
\hline & & & & $\mathrm{Ni}$ & $\mathrm{SiO}_{2}$ & $\mathrm{Fe}_{2} \mathrm{O}_{3}$ & $\mathrm{MgO}$ & $\mathrm{CO}$ \\
\hline 23 & 5438.197 & 9951.9 & 2812.5 & 0.92 & 43.74 & 16.52 & 12.5 & 0.01 \\
\hline 24 & 5026.23 & 9198 & 0.0 & 1.04 & 45.64 & 13.02 & 13.5 & 0.02 \\
\hline 25 & 17374.99 & 31796.24 & 1068 & 1.46 & 36.16 & 35.85 & 15.00 & 0.05 \\
\hline 26 & 22862.28 & 41837.97 & 0.0 & 1.42 & 39.97 & 28.62 & 11.20 & 0.04 \\
\hline 27 & 16356.25 & 29931.93 & 6142.75 & 1.62 & 38.85 & 34.08 & 9.80 & 0.06 \\
\hline 28 & 15587.5 & 28525.12 & 29875 & 1.57 & 42.51 & 23.61 & 7.98 & 0.02 \\
\hline 29 & 23640.62 & 43262.33 & 3625 & 1.20 & 48.8 & 24.08 & 15.6 & 0.05 \\
\hline 30 & 18418.72 & 33706.26 & 3406.25 & 1.20 & 45.6 & 17.13 & 20.1 & 0.04 \\
\hline 31 & 14018.71 & 25654.24 & 4025 & 1.60 & 45.4 & 29.81 & 7.50 & 0.05 \\
\hline 32 & 20906.24 & 38258.42 & 3465.5 & 1.56 & 38.1 & 20.07 & 5.6 & 0.03 \\
\hline 33 & 14100 & 25803 & 11106.5 & 1.21 & 56.2 & 22.79 & 13.2 & 0.01 \\
\hline 34 & 14108.11 & 25817.84 & 11693.15 & 0.96 & 55.1 & 14.26 & 24.00 & 0.01 \\
\hline 35 & 9249.989 & 16927.48 & 11802.7 & 1.24 & 54.2 & 17.93 & 7.80 & 0.01 \\
\hline 36 & 9856.213 & 18036.87 & 7624.7 & 1.75 & 53.4 & 20.01 & 9.20 & 0.050 \\
\hline 37 & 7681.831 & 14057.75 & 9924.9 & 1.40 & 48.1 & 21.86 & 10.2 & 0.05 \\
\hline 38 & 12143.72 & 22223 & 3606.2 & 0.99 & 50.9 & 21.74 & 8.5 & 0.06 \\
\hline 39 & 15855.03 & 29014.7 & 2487.5 & 1.23 & 50.1 & 21.35 & 15.2 & 0.05 \\
\hline 40 & 12743.72 & 23321 & 3349.9 & 1.51 & 56.5 & 20.14 & 8.05 & 0.03 \\
\hline 41 & 1999.945 & 3659.9 & 9005 & 0.76 & 54.7 & 21.46 & 7.09 & 0.01 \\
\hline 42 & 5793.716 & 10602.5 & 0.0 & 0.92 & 55.8 & 16.80 & 8.5 & 0.02 \\
\hline Total & 263162 & 481586.5 & 125020.60 & 1.23 & 46.60 & 23.31 & 12.06 & 0.04 \\
\hline
\end{tabular}

Table 4. Exploitable reserves above the 574 meters level

\begin{tabular}{ccccccccc}
\hline \multirow{2}{*}{ Level } & \multirow{2}{*}{${\text { Sterile, } \mathrm{m}^{3}}^{*}$ Ore, $\mathrm{m}^{3}$} & \multirow{2}{*}{ Ore, $\mathrm{s}$} & \multicolumn{5}{c}{ Quality at source, \% } \\
\cline { 5 - 9 } & & & $\mathrm{Ni}$ & $\mathrm{SiO}_{2}$ & $\mathrm{Fe}_{2} \mathrm{O}_{3}$ & $\mathrm{MgO}$ & $\mathrm{CO}$ \\
\hline 595 & 8304 & 40755.98907 & 74583.46 & 1.12 & 46.22 & 14.2 & 11.40 & 0.02 \\
588 & 36792 & 126726.9508 & 231910.3 & 1.06 & 50.25 & 19.0 & 15.14 & 0.01 \\
581 & 102785.1 & 283702.45 & 519175.5 & 1.36 & 40.54 & 20.07 & 9.73 & 0.037 \\
574 & 125020.6 & 263161.9945 & 481586.5 & 1.23 & 46.60 & 23.31 & 12.06 & 0.04 \\
Total & 272901.7 & 714347.39 & 1307256 & 1.25 & 44.82 & 20.74 & 11.64 & 0.03 \\
\hline
\end{tabular}

Finally, during the exploitations, we have the maximal height of the level (floor) 7 meters, minimal $0 \mathrm{~m}$, ore amount for the volume weight is $1.83 \mathrm{t} / \mathrm{m}^{3}$ and the sterile-ore no balance/ore ratio is $0.38: 1 \mathrm{~m}^{3} / \mathrm{m}^{3}$.
The exploitation duration depends on the amount of exploitable reserves and annual production plan, which is determined according to the following formula: 


$$
T=\frac{Q_{r e}}{Q_{p v}}=\frac{1307256}{605793}=2.15 \text { years. }
$$

For the annual production of 605793 t/year, this amount of exploitable reserves guarantees an exploitation period of 2.15 years.

$Q_{\text {re }}$, exploitable reserves, tons.

$Q_{p v}$, annual production, tons.

Average nickel percentage and accompanying components: $\mathrm{Ni}=1.29 \%, \mathrm{SiO}_{2}=44.82 \%, \mathrm{Fe}_{2} \mathrm{O}_{3}=20.74 \%$, $\mathrm{MgO}=11.64 \%, \mathrm{Co}=0.03 \%$.

\section{Results and discussion}

In the mineral deposit of nickel silicate ore "Glavica" through Surpac software the deposit limit has been determined and reserves have been calculated according to the exploration drilling network $25 \times 25$ meters. The most important part here is to define the ore body geometry, evaluate the depth by drilling, inserting field data into the Surpac database i.e. assay, collar, and survey file as presented in Tables 5-7.

The Assay.csv database is presented in Table 5 describing the geological drilling (hole id) for each meter of depth of this drilling from the initial drilling quota (zero meters quota) to the final quota where the drilling at a depth of $33.2 \mathrm{~m}$ has been completed.

Table 5. Databases Assay.csv

\begin{tabular}{|c|c|c|c|c|c|c|c|c|}
\hline Hole id & From & To & $\begin{array}{l}\text { Lith_ } \\
\text { general }\end{array}$ & $\mathrm{Ni}$ & Co & $\mathrm{Fe}$ & $\mathrm{SiO}_{2}$ & Mgo \\
\hline 10 & 0 & 1 & $\mathrm{CY}$ & 0.08 & 0.04 & 15.09 & 68.18 & 0.73 \\
\hline 10 & 1 & 2 & $\mathrm{CY}$ & 0.04 & 0.03 & 11.87 & 68.44 & 3.59 \\
\hline 10 & 2 & 3 & CY & 0.08 & 0.03 & 11.87 & 68.44 & 3.59 \\
\hline 10 & 3 & 4 & CY & 0.13 & 0.03 & 11.87 & 68.44 & 3.59 \\
\hline 10 & 4 & 5 & CY & 0.2 & 0.01 & 19.14 & 52.54 & 1.38 \\
\hline 10 & 5 & 6 & CY & 0.31 & 0.01 & 19.14 & 52.54 & 1.38 \\
\hline 10 & 6 & 7 & CY & 0.15 & 0.02 & 33.12 & 40.63 & 5.78 \\
\hline 10 & 7 & 8.5 & CY & 0.2 & 0.04 & 15.09 & 68.18 & 0.73 \\
\hline 10 & 8.5 & 10.5 & CY & 0.15 & 0.04 & 15.09 & 68.18 & 0.73 \\
\hline 10 & 10.5 & 11.9 & CY & 0.29 & 0.01 & 19.14 & 52.54 & 1.38 \\
\hline 10 & 11.9 & 12.9 & CY & 0.21 & 0.03 & 11.87 & 68.44 & 3.59 \\
\hline 10 & 12.9 & 13.9 & $\mathrm{CY}$ & 0.27 & 0.02 & 10.53 & 63.01 & 16.89 \\
\hline 10 & 13.9 & 14.9 & $\mathrm{CY}$ & 0.43 & 0.04 & 13.88 & 75.44 & 3.7 \\
\hline 10 & 14.9 & 16.1 & $\mathrm{CY}$ & 0.25 & 0 & 14.66 & 77.6 & 0 \\
\hline 10 & 16.1 & 18.1 & $\mathrm{CY}$ & 0.31 & 0.12 & 32.56 & 54.78 & 0.46 \\
\hline 10 & 18.1 & 19.7 & $\mathrm{CY}$ & 0.08 & 0.01 & 19.14 & 52.54 & 1.38 \\
\hline 10 & 19.7 & 20.7 & $\mathrm{CY}$ & 0.18 & 0.05 & 36.63 & 41.91 & 3.7 \\
\hline 10 & 20.7 & 21.7 & $X$ & 0.82 & 0.03 & 28.75 & 56.32 & 4.48 \\
\hline 10 & 21.7 & 22.7 & $X$ & 1.02 & 0.11 & 39.8 & 39.73 & 3.01 \\
\hline 10 & 22.7 & 23.7 & $X$ & 1.99 & 0.06 & 26.95 & 50.74 & 5.01 \\
\hline 10 & 23.7 & 25 & $X$ & 2.62 & 0.05 & 22.4 & 61.17 & 3.7 \\
\hline 10 & 25 & 26.5 & $\mathrm{X}$ & 2 & 0.04 & 25.56 & 51.49 & 5.06 \\
\hline 10 & 26.5 & 27.2 & CY & 0.18 & 0.05 & 36.63 & 41.91 & 3.7 \\
\hline 10 & 27.2 & 31.9 & $X$ & 1.74 & 0.08 & 44.09 & 42.52 & 0 \\
\hline 10 & 31.9 & 33.2 & SPN & 0.35 & 0.05 & 33.44 & 39.42 & 5.24 \\
\hline 11 & 0 & 5.9 & $\mathrm{CY}$ & 0 & 0.03 & 17.43 & 42.34 & 21.72 \\
\hline 11 & 5.9 & 6.9 & $\mathrm{CY}$ & 0.24 & 0.02 & 12.26 & 40.87 & 16.6 \\
\hline 11 & 6.9 & 7.9 & $\mathrm{CY}$ & 0.42 & 0.02 & 11.27 & 40.55 & 29.79 \\
\hline 11 & 7.9 & 8.9 & $\mathrm{CY}$ & 0.6 & 0.02 & 15.42 & 40.16 & 22.32 \\
\hline 11 & 8.9 & 10.9 & $\mathrm{CY}$ & 0.44 & 0.06 & 53.02 & 28.47 & 4.12 \\
\hline 11 & 10.9 & 11.6 & CY & 0.51 & 0.07 & 57.59 & 21.55 & 2.16 \\
\hline 11 & 11.6 & 12.3 & X & 1.05 & 0.06 & 45.16 & 33.44 & 4.24 \\
\hline 11 & 12.3 & 13.3 & CY & 0.66 & 0.06 & 53.02 & 28.47 & 4.12 \\
\hline 11 & 13.3 & 14.3 & $X$ & 1.07 & 0.05 & 37.25 & 51.46 & 6.35 \\
\hline
\end{tabular}

For each depth of each drill in the Assay.csv file are placed the depths of each geological layer, the depths of the location of the source as well as the quality of nickel and other metallic elements which are present in the nickel ore source (Table 5).

The Collar.scv database describes the maximum depth of each drill as well as the geodetic coordinates $x, y$ and $z$ for each geological drill (Table 6).

\section{Table 6. Databases Collar.scv}

\begin{tabular}{|c|c|c|c|c|c|}
\hline Hole_id & Hole_path & Max_depth & $X$ & $Y$ & $\mathrm{Z}$ \\
\hline 10 & Linear & 33.2 & 7500948 & 4711746 & 560.21 \\
\hline 11 & Linear & 35.3 & 7500993 & 4711767 & 553.22 \\
\hline 13 & Linear & 59.2 & 7500878 & 4711659 & 544.41 \\
\hline 21 & Linear & 74 & 7501084 & 4711808 & 498.59 \\
\hline 25 & Linear & 28 & 7500791 & 4711729 & 576.83 \\
\hline 26 & Linear & 29.5 & 7501144 & 4711617 & 588.4 \\
\hline 29 & Linear & 32 & 7501018 & 4711833 & 545.03 \\
\hline 30 & Linear & 70 & 7501064 & 4711854 & 498.52 \\
\hline 31 & Linear & 55 & 7500857 & 4711704 & 547.89 \\
\hline 37 & Linear & 57 & 7501039 & 4711787 & 526.22 \\
\hline 68 & Linear & 50.5 & 7500938 & 4711467 & 558.78 \\
\hline 69 & Linear & 43.5 & 7500984 & 4711487 & 554.58 \\
\hline 77 & Linear & 35 & 7500840 & 4711862 & 568.1 \\
\hline 83 & Linear & 45 & 7500955 & 4711611 & 554.72 \\
\hline 107 & Linear & 24 & 7500770 & 4711774 & 580.62 \\
\hline 108 & Linear & 43 & 7500952 & 4711858 & 541.89 \\
\hline 1001 & Linear & 10.8 & 7500906 & 4711177 & 609.93 \\
\hline 1002 & Linear & 10 & 7500929 & 4711187 & 606.59 \\
\hline 1003 & Linear & 13.4 & 7500951 & 4711198 & 613.19 \\
\hline 1004 & Linear & 13.3 & 7500974 & 4711208 & 610.17 \\
\hline 1005 & Linear & 22 & 7500997 & 4711218 & 596.02 \\
\hline 1007 & Linear & 24 & 7500986 & 4711241 & 600.98 \\
\hline 1008 & Linear & 44 & 7500964 & 4711231 & 577.3 \\
\hline 1009 & Linear & 50 & 7500941 & 4711220 & 574.49 \\
\hline 1010 & Linear & 34 & 7500918 & 4711210 & 582.6 \\
\hline 1011 & Linear & 27.4 & 7500895 & 4711200 & 593.84 \\
\hline 1012 & Linear & 12.3 & 7500873 & 4711189 & 598.6 \\
\hline 1014 & Linear & 7 & 7500839 & 4711201 & 603.55 \\
\hline 1015 & Linear & 13.5 & 7500862 & 4711212 & 601.65 \\
\hline 1016 & Linear & 32 & 7500885 & 4711222 & 584.5 \\
\hline 1017 & Linear & 30 & 7500908 & 4711233 & 590.84 \\
\hline 1018 & Linear & 41.3 & 7500930 & 4711243 & 582.36 \\
\hline 1019 & Linear & 62 & 7500953 & 4711254 & 557.81 \\
\hline 1020 & Linear & 20 & 7500976 & 4711264 & 596.62 \\
\hline 1021 & Linear & 20 & 7500999 & 4711274 & 591.18 \\
\hline 1022 & Linear & 23 & 7501021 & 4711285 & 587.84 \\
\hline 1025 & Linear & 29 & 7501011 & 4711307 & 590.37 \\
\hline
\end{tabular}

The Survey.scv database describes all geological drilling specifying the name of each drill (Hole ID), their depth, drilling angle and drilling azimuth (Table 7).

Using Survey.scv, Collar.scv and Assay.csv databases by applying computer software we have managed to discretize (divide into mini-blocks) the source according to the real state in which the source is located.

After creation of excel database for 3D modeling purposes the ore body of employing Surpac software as shown in (Figs. 7 and 8). In (Fig. 8) is shown the ore body and is clearly seen the contact between footwall and hanging wall surrounding the ore body. The minerals thickness at the source ranges from 2-32 meters, the average thickness is about 11 meters. The source was formed in specific conditions during the formation process of the lateritic crust of the ultrabasic rocks' alienation. The following zones are distinguished in the "Gllavica" source in the downward direction (the source stratigraphy): the red to yellow clay zone, siliceous-opal zone, nontronite clay zone, serpentinized and nontronitized harzburgite zone, and fresh peridotites zone. 
Table 7. Databases Survey.scv

\begin{tabular}{|c|c|c|c|c|}
\hline $\mathrm{Ndx}$ & Hole-Id & Depth & Dip & Azimuth \\
\hline 1 & 10 & 33.2 & -90 & 0 \\
\hline 2 & 11 & 35.3 & -90 & 0 \\
\hline 3 & 13 & 59.2 & -90 & 0 \\
\hline 4 & 21 & 74 & -90 & 0 \\
\hline 5 & 25 & 28 & -90 & 0 \\
\hline 6 & 26 & 29.5 & -90 & 0 \\
\hline 7 & 29 & 32 & -90 & 0 \\
\hline 8 & 30 & 70 & -90 & 0 \\
\hline 9 & 31 & 55 & -90 & 0 \\
\hline 10 & 37 & 57 & -90 & 0 \\
\hline 11 & 68 & 50.5 & -90 & 0 \\
\hline 12 & 69 & 43.5 & -90 & 0 \\
\hline 13 & 77 & 35 & -90 & 0 \\
\hline 14 & 83 & 45 & -90 & 0 \\
\hline 15 & 107 & 24 & -90 & 0 \\
\hline 16 & 108 & 43 & -90 & 0 \\
\hline 17 & 1001 & 10.8 & -90 & 0 \\
\hline 18 & 1002 & 10 & -90 & 0 \\
\hline 19 & 1003 & 13.4 & -90 & 0 \\
\hline 20 & 1004 & 13.3 & -90 & 0 \\
\hline 21 & 1005 & 22 & -90 & 0 \\
\hline 22 & 1007 & 24 & -90 & 0 \\
\hline 23 & 1008 & 44 & -90 & 0 \\
\hline 24 & 1009 & 50 & -90 & 0 \\
\hline 25 & 1010 & 34 & -90 & 0 \\
\hline 26 & 1011 & 27.4 & -90 & 0 \\
\hline 27 & 1012 & 12.3 & -90 & 0 \\
\hline 28 & 1014 & 7 & -90 & 0 \\
\hline 29 & 1015 & 13.5 & -90 & 0 \\
\hline 30 & 1016 & 32 & -90 & 0 \\
\hline 31 & 1017 & 30 & -90 & 0 \\
\hline 32 & 1018 & 41.3 & -90 & 0 \\
\hline 34 & 1019 & 62 & -90 & 0 \\
\hline 35 & 1020 & 20 & -90 & 0 \\
\hline 36 & 1021 & 20 & -90 & 0 \\
\hline 37 & 1022 & 23 & -90 & 0 \\
\hline 38 & 1025 & 29 & -90 & 0 \\
\hline 39 & 1026 & 33 & -90 & 0 \\
\hline
\end{tabular}

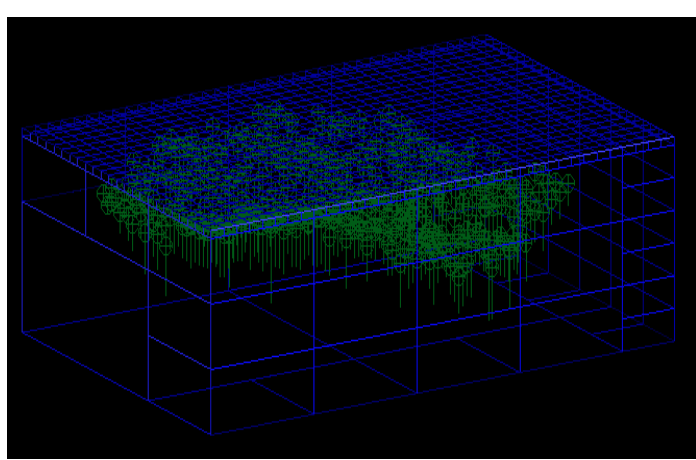

Figure 7. Geological drilling with different depths in each descreted block meeting the ore shale and geological contacts

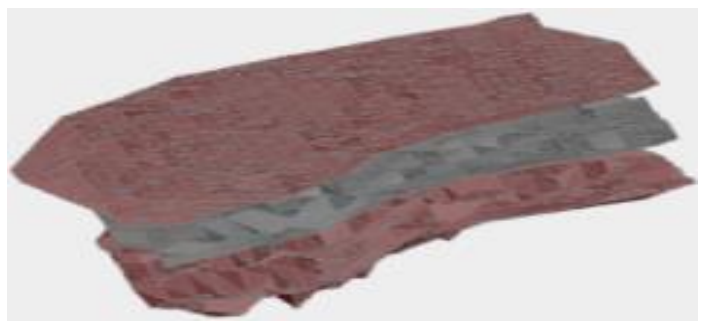

Figure 8. The definition of the nickel ore body layer is presented in gray and is located between the two clay layers - the ceiling contact and the floor contact
The databases were organized in Microsoft Excel by constructing tables with such structures so that they would be more useful for easier processing by the appropriate software. With the appropriate software first the data was processed, the isohypses of the field surface, isohypses of the ore ceiling and isohypses of the floor, profile construction, blocks and calculation of reserves.

After managing to divide the Gllavica source in mini blocks we can mix the blocks and at the same time we have tracked their exploitation and compliance of the data with the situation in the field. From the practical sense of the presentations of reserves and resources of polymetallic ore, it is of interest to have a table presentation of the ore amount (tonnage) on one side, and the quality on the other side.

\section{Conclusions}

The discretization of nickel source in $25 \times 25 \mathrm{~m}$ and $50 \times 50 \mathrm{~m}$ mini blocks, through deep geological drills has professionally enabled the correct assessment of the silicate ore quality in the entire source. Geological drills of each block have defined the ceiling and floor contacts of the ore body and have also defined the phenomena of the interposition of clay layers within the ore body.

Through transverse geological profiles the surface nickel exploitation intensity according to 595, 588, 581 and 574 m block levels has been addressed with special scientific dedication.

During the projection (study) phase the average nickel and accompanying components percentage was defined (assessed) in each level starting from $595 \mathrm{~m}: \mathrm{Ni}=1.12 \%, \mathrm{SiO}_{2}=46.22 \%$, $\mathrm{Fe}_{2} \mathrm{O}_{3}=14.20 \%, \mathrm{MgO}=11.40 \%, \mathrm{Co}=0.05 \%$; $588 \mathrm{~m}$ level: $\mathrm{Ni}=1.06 \%, \mathrm{SiO}_{2}=50.25 \%, \mathrm{Fe}_{2} \mathrm{O}_{3}=19.00 \%, \mathrm{MgO}=15.14 \%$, $\mathrm{Co}=0.012 \% ; 581 \mathrm{~m}$ level: $\mathrm{Ni}=1.36 \%, \quad \mathrm{SiO}_{2}=40.54 \%$, $\mathrm{Fe}_{2} \mathrm{O}_{3}=20.07 \%, \mathrm{MgO}=9.73 \%, \mathrm{Co}=0.037 \%$, and $574 \mathrm{~m}$ level with the following accompanying components: $\mathrm{Ni}=1.34 \%, \mathrm{SiO}_{2}=46.60 \%, \mathrm{Fe}_{2} \mathrm{O}_{3}=23.31 \%, \mathrm{MgO}=12.06 \%$, $\mathrm{Co}=0.04 \%$.

Based on the ore reserves of 1307256.00 tons and production demands of 605793 [t/year] the mine longevity is expected to be 2.15 years, and the average nickel and accompanying components percentage in the entire source is: $\mathrm{Ni}=1.29 \%, \mathrm{SiO}_{2}=44.82 \%, \mathrm{Fe}_{2} \mathrm{O}_{3}=20.74 \%, \mathrm{MgO}=11.64 \%$, $\mathrm{Co}=0.03 \%$.

\section{Acknowledgements}

I had the honor and pleasure to contribute in this paper, which was completed thanks to the deep cooperation with the professional staff of the mine with whom we contributed jointly so that this paper would provide the best possible assessment for the managing and engineering staff that shall implement this study in the mine circumstances.

\section{References}

[1] Zeqiri, R. (2020). Geostatistical analysis of the nickel source in Gllavica mine, Kosovo. Mining of Mineral Deposits, 14(2), 53-58. https://doi.org/10.33271/mining14.02.053

[2] Materiali, M. (2004). Arkivi i minierës së gllavicës. "Ferronikel".

[3] Zeqiri, R. (2012). Geostatistics in modern mining planning. Journal of International Environmental Application and Science, 7(2), 310-317.

[4] Rafet, Z., Jahir, G., Muhamedin, H., \& Gzim, I. (2016). Distribution of valuable metals in various horizons of "Trepça" mine. Journal International Application \& Science, 11(4), 346-350.

[5] Kelmendi, Sh., \& Zeqiri, I. (2006). Mathematical methods in engineering. Pristina, Kosovo: University of Prishtina. 
[6] Pariseau, G.E. (2007). Design analysis in rock mechanics. London, United Kingdom: Taylor \& Francis Group. https://doi.org/10.1201/9780203968253

[7] Zeqiri, R., Riheb, H., Karim, Z., Younes, G., Rania, B., \& Aniss, M. (2019). Analysis of safety factor of security plates in the mine "Trepça" Stantërg. Mining Science, (26), 21-36. https://doi.org/10.37190/msc192602

[8] Zeqiri, R.R., Gashi, J., \& Kutllovci, F. (2019). Stability analysis of security pillars with dimension $10 \times 10 \mathrm{~m}$ fromed by ore of mineral body during the exploitation of the "Trepça" mine in Stantërg. Mining Science, (26), 37-44. https://doi.org/10.37190/msc192603

[9] Zeqiri, I. (2012). Ddesigning of new underground mining facilities with dual function in the $\mathrm{Pb}-\mathrm{Zn}$ mine of Mazhiq. SGEM2012 $12^{\text {th }} \mathrm{In}$ ternational Multidisciplinary Scientific GeoConference, (1), 523-530. https://doi.org/10.5593/sgem2012/s03.v1016
[10] Zeqiri, I., Gashi, J., \& Zeqiri, R. (2012). Designing of new underground mining facilities with dual function in the $\mathrm{Pb}-\mathrm{Zn}$ mine of Mazhiq. Ecology, Economics, Education and Legislation, (1).

[11] Zeqiri, I. (2011). The impact of arming methods in security scale during the application of frontal methods in mine Trepca in Stanterg. SGEM2011 11th International Multidisciplinary Scientific GeoConference. https://doi.org/10.5593/sgem2011/s03.145

[12] Zeqiri, I. (2011). The exploitation system of securing backbone in upper levels - Trepca mine, Stanterg. SGEM2011 11th International Multidisciplinary Scientific GeoConference, https://doi.org/10.5593/sgem2011/s03.144

[13] Kelmendi, S. (2012). Decomposition of flotation process precondition for mathematical modeling. SGEM2012 12th International Multidisciplinary Scientific GeoConference. https://doi.org/10.5593/sgem2012/s04.v2002

\section{Дискретизація та оцінка якості нікелю на шахті “Главиця”, Косово}

\section{Р. Зекірі}

Мета. Моделювання геологічної будови рудного тіла, що представляє промисловий інтерес для високоточної якісно-кількісної оцінки родовища нікелю на шахті “Главиця".

Методика. 3 огляду на інтенсивний розвиток професійного програмного забезпечення в області вирішення завдань гірничорудної промисловості, на шахті “Главиця” була використана програма Surpac v.6.2, яка дозволяє провести якісну та кількісну оцінку покладів нікелю в Косово. Використувалися дані дослідницьких бурових робіт зі щільністю сітки $25 \times 25$ метрів на довжині 125 м, а потім сітка буріння склала $50 \times 50$ метрів.

Результати. Грунтуючись на даних геологічної розвідки, буріння та взяття зразків породи, було визначено якість руди в лежачому і висячому боках рудного покладу. За допомогою Surpac v.6.2 був досліджений клас руди в контексті поздовжнього геологічного профілю, для чого дані по кожній пробуреній свердловині були оцифровані та перетворені у файли для кількісного аналізу зразків, файли для знаходження мінімуму/максимуму і маркшейдерські файли. Визначено очікуваний термін служби рудника 2.15 років з урахуванням запасів руди 1.307 млн т і рівня виробничих потреб в 605.7 тис. т/рік, а також встановлено середній відсоток нікелю і супутніх компонентів у родовищі, який становить: $\mathrm{Ni}=1.29 \%, \mathrm{SiO}_{2}=44.82 \%, \mathrm{Fe}_{2} \mathrm{O}_{3}=20.74 \%, \mathrm{MgO}=11.64 \%, \mathrm{Co}=0.03 \%$.

Наукова новизна. Надана високоякісна оцінка родовища нікелю в умовах шахти "Главиця" на основі застосування адекватного програмного забезпечення, створення баз даних у відповідності зі значеннями, отриманими при натурних геологічних вимірах.

Практична значимість. Геологічна розвідка забезпечує безпечну розробку рудників протягом всього періоду їх експлуатації і дозволяє відокремити породні блоки, які відрізняються від інших в плані якості руди. Таким чином, дискретизація покладу дозволила нам оцінити реальну якість і кількість руди в порівнянні з даними, отриманими методом профілізації.

Ключові слова: рудник, нікель, вміст у руді, розробка, моделювання

\section{Дискретизация и оценка качества никеля на шахте "Главица", Косово}

\section{Р. Зекири}

Цель. Моделирование геологического строения рудного тела, представляющего промышленный интерес для высокоточной качественно-количественной оценки месторождения никеля на шахте “Главица".

Методика. Учитывая интенсивное развитие профессионального программного обеспечения в области решения задач горнорудной промышленности, на шахте “Главица" была использована программа Surpac v.6.2, которая позволяет произвести качественную и количественную оценку залежей никеля в Косово. Использовались данные исследовательских буровых работ с плотностью сетки $25 \times 25$ метров на длине 125 м, а затем сетка бурения составила $50 \times 50$ метров.

Результаты. Основываясь на данных геологической разведки, бурения и взятия образцов породы, было определено качество руды в лежачем и висячем боках рудной залежи. При помощи Surpac v.6.2 был исследован класс руды в контексте продольного геологического профиля, для чего данные по каждой пробуренной скважине были оцифрованы и преобразованы в файлы для количественного анализа образцов, файлы для нахождения минимума/максимума и маркшейдерские файлы. Определен ожидаемый термин службы рудника - 2.15 лет с учетом запасов руды 1.307 млн т и уровня производственных потребностей в 605.7 тыс. т/год, а также установлен средний процент никеля и сопутствующих компонентов в месторождении, который составляет: Ni $=1.29 \%$, $\mathrm{SiO}_{2}=44.82 \%, \mathrm{Fe}_{2} \mathrm{O}_{3}=20.74 \%, \mathrm{MgO}=11.64 \%, \mathrm{Co}=0.03 \%$

Научная новизна. Дана высококачественная оценка месторождения никеля в условиях шахты “Главица" на основе применения адекватного программного обеспечения, создание баз данных в соответствии со значениями, полученными при натурных геологических измерениях.

Практическая значимость. Геологическая разведка обеспечивает безопасную разработку рудников в течение всего периода их эксплуатации и позволяет отделить породные блоки, которые отличаются от других в плане качества руды. Таким образом, дискретизация залежи позволила нам оценить реальное качество и количество руды в сравнении с данными, полученными методом профилизации.

Ключевые слова: рудник, никель, содержание в руде, разработка, моделирование 